This item was submitted to Loughborough's Research Repository by the author.

Items in Figshare are protected by copyright, with all rights reserved, unless otherwise indicated.

\title{
Baseline adaptive wavelet thresholding technique for sEMG denoising
}

PLEASE CITE THE PUBLISHED VERSION

http://dx.doi.org/10.1063/1.3596644

\section{PUBLISHER}

(c) American Institute of Physics

\section{VERSION}

VoR (Version of Record)

\section{LICENCE}

CC BY-NC-ND 4.0

\section{REPOSITORY RECORD}

Bartolomeo, Luca, Massimiliano Zecca, Salvatore Sessa, Zhuohua Lin, Y. Mukaeda, Hiroyuki Ishii, and Atsuo Takanishi. 2019. "Baseline Adaptive Wavelet Thresholding Technique for Semg Denoising". figshare. https://hdl.handle.net/2134/17576. 


\section{AIP Conference Proceedings}

Baseline Adaptive Wavelet Thresholding Technique for sEMG Denoising

L. Bartolomeo, M. Zecca, S. Sessa, Z. Lin, Y. Mukaeda et al.

Citation: AIP Conf. Proc. 1371, 205 (2011); doi: 10.1063/1.3596644

View online: http://dx.doi.org/10.1063/1.3596644

View Table of Contents: http://proceedings.aip.org/dbt/dbt.jsp?KEY=APCPCS\&Volume=1371\&lssue=1

Published by the American Institute of Physics.

\section{Additional information on AIP Conf. Proc.}

Journal Homepage: http://proceedings.aip.org/

Journal Information: http://proceedings.aip.org/about/about_the_proceedings

Top downloads: http://proceedings.aip.org/dbt/most_downloaded.jsp?KEY=APCPCS

Information for Authors: http://proceedings.aip.org/authors/information_for_authors

- Article-level metrics now available 


\title{
Baseline Adaptive Wavelet Thresholding Technique for sEMG Denoising
}

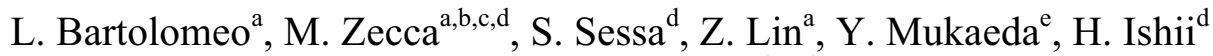 \\ and Atsuo Takanishi ${ }^{\mathrm{a}, \mathrm{b}, \mathrm{c}, \mathrm{d}}$
}

\author{
${ }^{a}$ Global Robot Academia, Waseda University, Tokyo, Japan \\ ${ }^{b}$ Humanoid Robotics Institute (HRI), Waseda University, Tokyo, Japan \\ 'Italy-Japan Joint Laboratory on Humanoid and Personal Robotics "RoboCasa", Tokyo, Japan \\ ${ }^{d}$ Faculty of Science and Engineering, Waseda University, Tokyo, Japan \\ ${ }^{e}$ Graduate School of Engineering, Waseda University, Tokyo, Japan
}

\begin{abstract}
The surface Electromyography (sEMG) signal is affected by different sources of noises: current technology is considerably robust to the interferences of the power line or the cable motion artifacts, but still there are many limitations with the baseline and the movement artifact noise. In particular, these sources have frequency spectra that include also the lowfrequency components of the sEMG frequency spectrum; therefore, a standard all-bandwidth filtering could alter important information. The Wavelet denoising method has been demonstrated to be a powerful solution in processing white Gaussian noise in biological signals. In this paper we introduce a new technique for the denoising of the sEMG signal: by using the baseline of the signal before the task, we estimate the thresholds to apply to the Wavelet thresholding procedure. The experiments have been performed on ten healthy subjects, by placing the electrodes on the Extensor Carpi Ulnaris and Triceps Brachii on right upper and lower arms, and performing a flexion and extension of the right wrist. An Inertial Measurement Unit, developed in our group, has been used to recognize the movements of the hands to segment the exercise and the pre-task baseline. Finally, we show better performances of the proposed method in term of noise cancellation and distortion of the signal, quantified by a new suggested indicator of denoising quality, compared to the standard Donoho technique.
\end{abstract}

Keywords: Wavelet Denoising, Surface Electromyography.

\section{INTRODUCTION}

Surface Electromyography (sEMG) provides a safe, easy and non-invasive method to quantify the energy of the muscles [1]: for these reasons sEMG is currently becoming more and more a powerful tool for the assessment in sport [2], [3], rehabilitation [4], [5], ergonomic design [6], [7], and medical robotics [8].

However, the sEMG is affected by various sources of noises, including the power line interference, the noise generated by the cable motion, the baseline and the movement artifact noise. The baseline is the combination of the two noise sources originated in the electronics of the amplification system (thermal noise) and at the skin-electrode interface (electrochemical noise), respectively [9], [10].

While the power line interference and the cable motion artifact can be removed

2011 International Symposium on Computational Models for Life Sciences (CMLS-11)

AIP Conf. Proc. 1371, 205-214 (2011); doi: 10.1063/1.3596644

(C) 2011 American Institute of Physics 978-0-7354-0931-6/\$30.00 
using standard filtering procedures [1], [11], the baseline and the movement artifact have spectra that include also the low frequency spectrum of the EMG signal and a standard filtering has the risk to alter important information of the signal. The solution would be to filter the maximum quantity of noise while keeping as much of the effective signal frequency spectrum as possible.

Wavelet denoising algorithms have been received extensive consideration in the processing of white Gaussian noise in biological signals, especially for the Electrocardiogram [12]-[14]. Most wavelet based denoising literatures suggest the use of the Donoho's method [15], [16], that makes an estimation of the thresholds by maximizing a risk function in terms of quadratic loss at the sample points. However, this method has limitations in the denoising of EMG signal, because it does not only remove noises but also significant part of signal with a consequent loss of geometrical characteristics of the signal. Different studies [17], [18] have proposed to change the thresholds by using different statistical techniques, with some improvements in the denoising, but still the results have not been completely confirmed.

In this study we propose a new method to denoise the sEMG signal by using the baseline to estimate the thresholds of the Wavelet denoising algorithm. The advantage of the sEMG is that the baseline could be taken before the exercise, in different conditions: Relax Task, when the subject is kept relaxed, or just before starting the designed exercise, Pre-Task, when the muscle is already under some tension.

Finally, we compare these results with the Donoho method to show better noise rejection during the noisy interval and the higher likelihood with the original signal during the exercise interval.

\section{WAVELET DENOISING ALGORITHM}

\section{A. Overview}

Given a general model of a noisy signal $s(n)=f(n)+e(n)$, the objective of the Wavelet Denoising Algorithm is to discard the noise part $e(n)$ of a signal $s(n)$ and to recover $f(n)$, with $e(n)$ to be considered usually a white Gaussian noise.

The procedure of wavelet denoising is composed of three steps.

At first the signal is decomposed by using the Stationary discrete Wavelet Transform (SWT) [19]. This first step requires the choice of the mother wavelet and the level of decomposition $N$. The details and approximation coefficients for each level are obtained.

The second step is the thresholding. For each level of decomposition the detail coefficients are compared with a level of threshold, then the signal is suppressed or transformed if it is smaller than the threshold. This second step requires the choice of the thresholds and the transformation function for the different levels of decomposition.

Finally, at the third step, the signal is reconstructed by applying the Inverse Stationary Wavelet Transform (ISWT) to the approximation coefficient at level $N$ and 
the modified detail coefficients from level 1 to $N$.

The summarized flow chart for the denoising algorithm is showed in Fig.1.

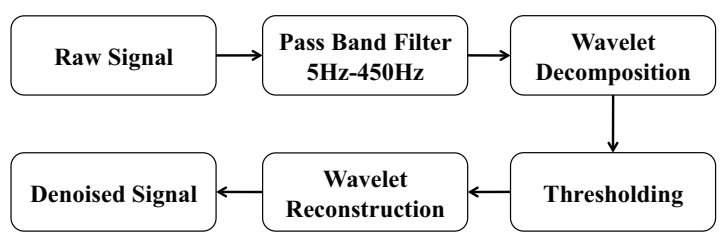

FIGURE 1. Flow chart of the Wavelet Denoising Algorithm.

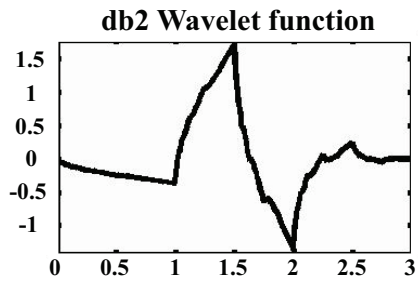

FIGURE 2. Wavelet function Daubechies $\mathrm{db} 2$

For this work we have considered the Daubechies $\mathrm{db} 2$ mother wavelet, presented in Fig.2, and the forth decomposition level, that have been proved, in case of myoelectric signals, to have the lowest mean square error [17].

\section{B. Thresholding}

The selection of the threshold with the universal threshold estimation method proposed by Donoho and Johnstone [15] is usually used as comparison to evaluate new techniques of denoising, due to its conservative nature [20]. Universal threshold estimation method uses a fixed value, $T H R_{U N I}=\sigma \sqrt{2 \log (N)}$ where $\mathrm{N}$ is the length of the samples of the time-domain signal and $\sigma$ is standard deviation of noise. The noise level $\sigma$ is estimated as the median of the absolute value of the detailed coefficients at the decomposition level $j$, divided by 0.6745 , a normalization factor.

Other thresholds have been introduced using different criteria as Minimax and SURE (Stain's Unbiased Risk Estimate), in which the universal threshold has been modified basically by multiplying a scale factor [21]-[24].

For each level of decomposition the detail coefficients are compared with the threshold, and then the signal is suppressed or transformed if it is smaller than the threshold: the common ways to modify the signal after the level comparisons are the hard and soft thresholding. In the Hard Thresholding (HT) the detail coefficient is completely suppressed if its absolute value is smaller than the threshold:

$$
c D_{j}= \begin{cases}c D_{j} & \text { if }\left|c D_{j}\right| \geq T H R_{j} \\ 0 & \text { otherwise }\end{cases}
$$

where the $T H R_{j}$ is the selected threshold at level $j$.

Differently, in the Soft Thresholding (ST) the signal is linearly shrunk as the following: 


$$
c D_{j}= \begin{cases}c D_{j}-T H R_{j} & \text { if } c D_{j} \geq T H R_{j} \\ 0 & \text { if }\left|c D_{j}\right|<T H R_{j} \\ c D_{j}+T H R_{j} & \text { if } c D_{j} \leq-T H R_{j}\end{cases}
$$

\section{EXPERIMENTAL SETUP}

The sEMG signals has been recorded from Extensor Carpi Ulnaris and Triceps Brachii of ten healthy volunteers, with age range 23-33 years old; we used a pair of surface DE-2.1 sensors (Delsys Inc.) and the signal were amplified by a BagnoliTM 16-channel system (Delsys Inc.) with a Gain $\mathrm{K}=100$. The skin was cleaned by mildly scrubbing it with 70\% isopropyl alcohol. The sensors were attached to the skin with a double-sided adhesive interface. The sEMG sensor was located in the midline of the muscle belly between the nearest innervation zone and the myotendinous [25]. A Dermatrodes HE-R (American Imex) electrode $(5.08 \mathrm{~cm}$ dia.) was located on the iliac crest to provide a reference. Sampling rate was set at 1000 samples per second using a 16-bit A/D converter board (National Instruments, USA, PCI-6034E). The acquisition software has been developed in $\mathrm{C}++$ and data have been processed using MATLAB 7.7 (R2008b). In order to have a synchronization between the movement of the hand and sEMG data, an Inertial Measurement Unit named WB-3, developed in our group, has been used: it is a 9-axis inertial module composed by a 3-axis accelerometer, 3axis gyroscope and 3-axis compass, extremely light, 2.9g. For this experiment, the gyroscope data have been considered [26].

The experimental protocol has been implemented by recording:

1. Relax Baseline (RB): it is the baseline when the volunteers were completely relaxed seated on a chair - relax task - for around 20 s.

2. Pre-Task Baseline (PTB): it is the baseline recorded with the volunteers keeping the arm extended in front of them, just before starting the task, for around $5 \mathrm{~s}$.

3. Exercise: the volunteers performed wrist extension for 5 times with $2 \mathrm{~s}$ intervals as shown in Fig. 3, for a total of $15 \mathrm{~s}$.

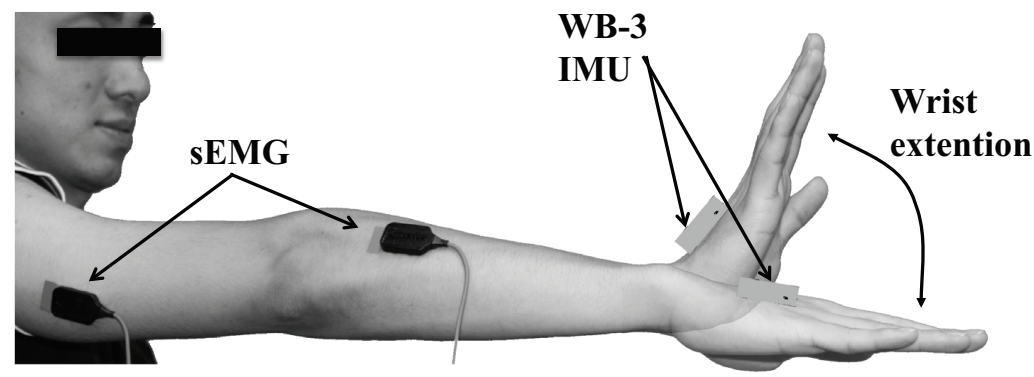

FIGURE 3. Exercise: the volunteer performed wrist extension for 5 times with 2 s intervals. 


\section{EVALUATION METHOD}

By using the information coming from the gyroscope of the Inertial Measurement Unit, an only-noise portion of the signal is separated from a task portion as showed in Fig.4. Indicating $n_{o}(n)$ the noise portion of the original signal, and $n_{d}(n)$ the noise portion after the denoising, they are compared with a defined Noise Ratio $(N R)$ by:

$$
N R=\frac{R M S\left(n_{d}(T)\right)}{R M S\left(n_{o}(T)\right)}, T \in \text { Baseline Portion }
$$

where the RMS indicates the root mean square and $\mathrm{T}$ is the interval of the baseline portion as in Fig.4. With this approach it is possible to have a number related only to the baseline segment of the signal and it is indicative of the noise cancellation. Smaller is this value, higher is the quality of the noise rejection.

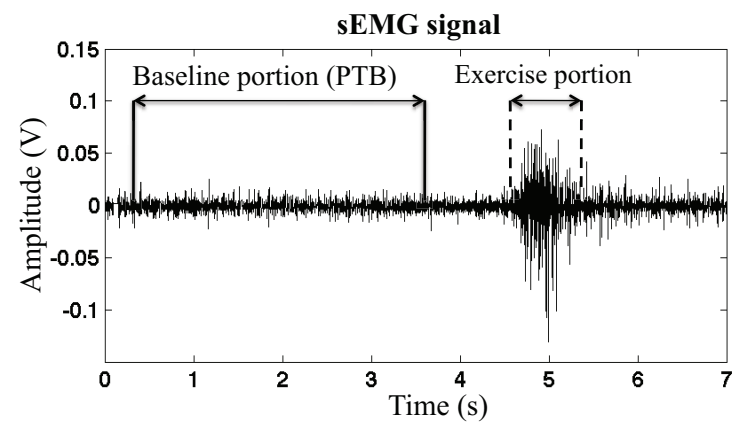

FIGURE 4. sEMG of Extensor Carpi Ulnaris. (a) Separation of the original signal in two segments containing the baseline, Baseline portion, and the Exercise portion.

In other way, denoting $t_{o}(n)$ the waveform of the task portion of the original signal and $t_{d}(n)$ the one after the denoising procedure, they are compared by using an error function that measures the misfit, point by point, of the two functions, given by the sum of the squares of the differences, divide by the RMS of $t_{o}(n)$ :

$$
E_{t}=\frac{1}{2} \sum_{j=1}^{N}\left(\frac{t_{o}(j)-t_{d}(j)}{R M S\left(t_{o}\right)}\right)^{2}
$$

where $\mathrm{N}$ is the number of the samples in the exercise portion. To facilitate the analysis, we consider the RMS value of $E_{t}$ :

$$
E R=\sqrt{\frac{2 E_{t}}{N}}
$$

where $\mathrm{N}$ is again the number of samples in the exercise portion and it is used to 
normalize the error in order to compare different sizes of signal in equal way. Smaller is the $E R$, smaller is the distortion due to the denoising algorithm.

We propose the combination of these two parameters to give an estimation of the denoising quality and to compare different methods.

$$
\begin{gathered}
D Q_{\%}=1-\left(\alpha_{N R} N R+\alpha_{E R} E R\right) \\
\alpha_{N R}+\alpha_{E R}=1
\end{gathered}
$$

where $D Q_{\%}$ indicates the quality of the denoising in percentage, $\alpha_{N R}$ is the weight of the noise ratio and $\alpha_{E R}$ is the weight of the distortion. For our evaluation we have chosen $\alpha_{N R}=0.7$ and $\alpha_{E R}=0.3$, giving more importance to the noise reduction than distortion; in any case, the proposed technique has showed better performances than Donoho standard method for all the combinations $\alpha_{N R}$ and $\alpha_{E R}$.

\section{BASELINE ADAPTIVE TECHNIQUE}

The Donoho method and its derivatives have been considered suitable for the denoising of sEMG, because the baseline has been approximated as a white Gaussian noise, distributed equally on the entire spectra of the signal. In case of Relax Baseline, this approach is valid, as showed in Fig.5: spectrum of the RB is uniformly distributed.

Our approach is to clean the signal from a baseline that is not a white Gaussian noise: in case of PTB, the arm is already under tension and the spectrum is not distributed equally, as showed in Fig.4 for the Extensor Carpi Ulnaris.

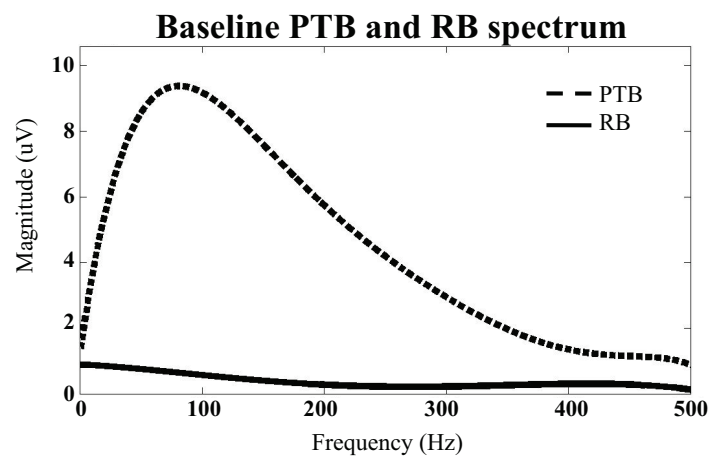

FIGURE 5. Extensor Carpi Ulnaris. Spectrum of the PTB and RB by Fast Fourier Transform of the waveform. The RB is equally distributed, while the PTB shows a complex distribution.

Based on this consideration, the thresholds will be derived in two ways:

1. Full-Baseline Adaptive (FBA): the thresholds are calculated in order to reduce, for each level of decomposition, the noise level down to 1\%. As shown in Fig.6, the algorithm keeps in memory the original RMS value of the detail coefficient, which is also the first Threshold applied to it. After, the RMS of the modified detailed is 
calculated, and if the new RMS is bigger than $1 \%$ of the original one, the threshold is updated by increasing of $10 \%$ respect to the previous value.

2. Semi-Baseline Adaptive (SBA): the thresholds are kept fixed at 3.09 times the RMS of the baseline for each level of decomposition. The choice of 3.09 considers the waveform approximated as gaussian, thus the $99.8 \%$ of the baseline noise will be cut: $\operatorname{erf}(3.09 / \sqrt{2})=0.998$. This is a strong hypothesis, because the PTB is not a Gaussian noise, as showed in Fig.5.

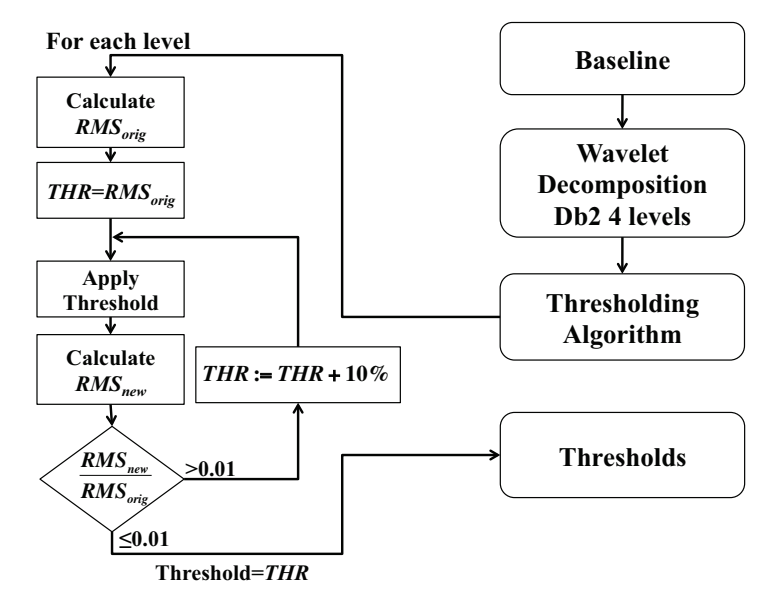

FIGURE 6. FBA algorithm. The thresholds are chosen in order to reduce the noise, for each level of decomposition, down to $1 \%$.

\section{RESULTS AND DISCUSSION}

The averaged results, together with the standard deviations, for the ten subjects for the Extensor Carpis Ulnaris are summarized in Table 1. The first column indicates the algorithm: FBA is the Full-Baseline Adaptive, SBA is the Semi-Baseline Adaptive and Donoho is the standard Donoho algorithm. The columns from second to fifth are respectively, the Denoise Quality calculate as in (6) in case of: 1. Relax Baseline used to choose the thresholds and applied Soft Thresholding (2), 2. Pre-Task Baseline and Soft Thresholding, 3. Relax Baseline and Hard Thresholding (1) and 4. Pre-Task Baseline and Hard Thresholding. In light gray it is highlighted the maximum value of the Denoise Quality, using the different algorithms and thresholds.

Table 1: Denoise Quality Results for the Extensor Carpi Ulnaris

Averaged Values for the ten subjects

\begin{tabular}{lcccc}
\hline Algorithm & $\begin{array}{c}\boldsymbol{D Q}_{\%} \\
\text { Relax Baseline } \\
\text { Soft Threshold }\end{array}$ & $\begin{array}{c}\text { Pre-Task Baseline } \\
\text { Soft Threshold }\end{array}$ & $\begin{array}{c}\boldsymbol{D Q}_{\%} \\
\text { Relax Baseline } \\
\text { Hard Threshold. }\end{array}$ & $\begin{array}{c}\boldsymbol{D Q}_{\%} \\
\text { Pre-Task Baseline } \\
\text { Hard Threshold }\end{array}$ \\
\hline FBA & $72.204 \pm 3.82$ & $75.320 \pm 3.18$ & $60.082 \pm 11.22$ & $82.362 \pm 2.54$ \\
SBA & $64.718 \pm 12.18$ & $75.070 \pm 3.52$ & $49.514 \pm 19.55$ & $81.269 \pm 2.19$ \\
Donoho & $71.107 \pm 3.01$ & $71.107 \pm 3.01$ & $71.526 \pm 3.87$ & $71.526 \pm 3.87$ \\
\hline
\end{tabular}


The results in Table 1 show that the FBA performs generally better, especially with the Pre-Task Baseline; moreover, the standard deviations of the FBA and SBA with the Pre-Task Baseline and Hard Thresholding are the smallest ones in terms of percentage, giving a very good statistics for the proposed algorithm. The Donoho method has the worst performance because it does not consider the baseline and makes a probabilistic estimation of the thresholds independently.

(a)

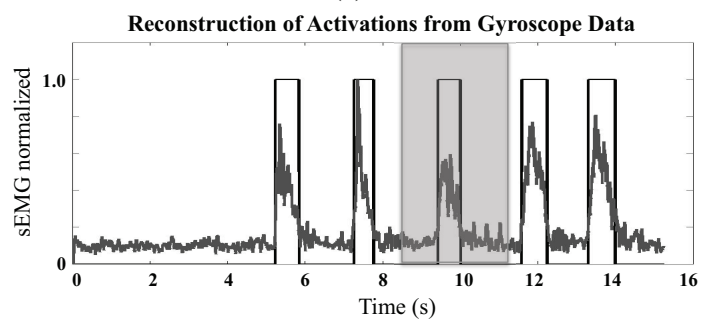

(c)

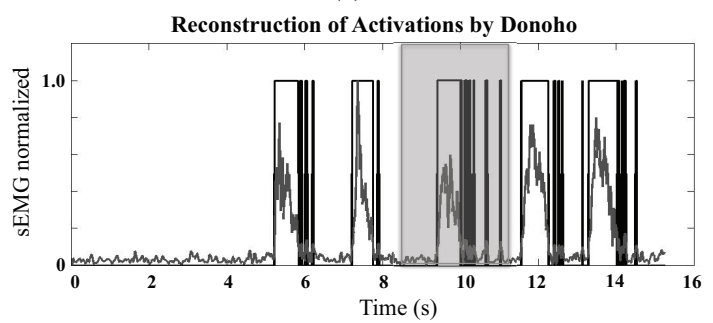

(e)

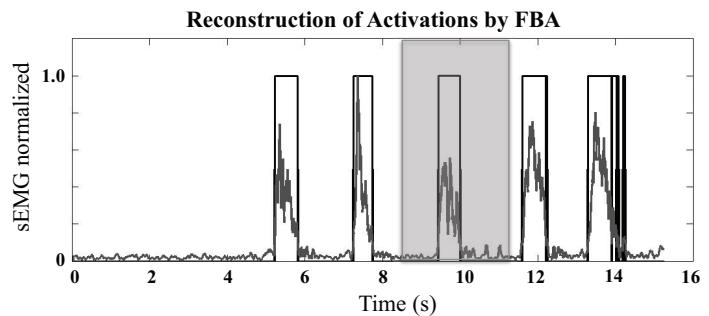

(b)

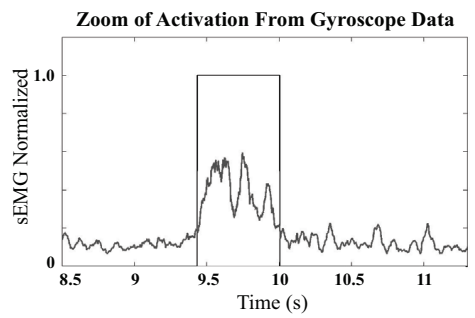

(d)

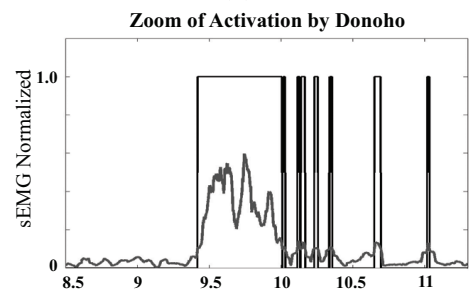

(f)

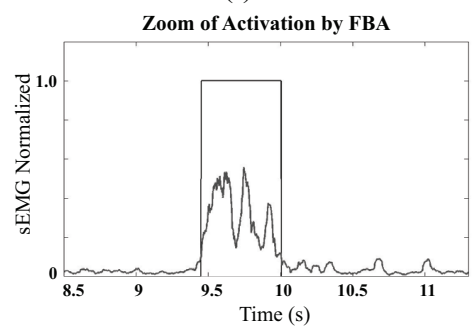

FIGURE 7. Normalized sEMG of Extensor Carpi Ulnaris. (a) Reconstruction of activations of muscles from motion data coming from Gyroscope. (b) Zoom of first activation from Gyroscope. (c) Reconstruction of activations by Donoho method (d) Zoom of first activation by threshold on/off with signal denoised by Donoho technique. (e) Reconstruction of activations by proposed FBA method (f) Zoom of first activation by threshold on/off with signal denoised by FBA algorithm.

Fig. 7 presents the comparison in term of reconstruction of activations between the sEMG of the Extensor Carpi Ulnaris denoised by Donoho standard method and by the proposed FBA, considering the data coming from the gyroscope as the ground. The activation has been calculated by comparing the windowed averaged signal (on a window of 50ms) with a fixed threshold, calculated as the mean of the signal during the first 3 seconds plus 5 times the standard deviation: if the signal is bigger than the threshold, the result is 1 , otherwise is 0 . In particular (c) is the normalized sEMG of 
the signal denoised by Donoho standard method with the reconstructed activations; (d) is the zoom of the gray part in (c), corresponding to the second attivation; (e) is the normalized sEMG of the signal denoised by the proposed FAB algorithm with the reconstructed activations; (f) is the zoom of the gray part of (e). It is evident that the proposed algorithm is performing better than the Donoho standard denoising, because it rejects the noise very well in portions of the signal when there are not movements and it is almost immune to false activations.

\section{CONCLUSIONS}

In this paper we have introduced a new technique to denoise a sEMG signal by using its baseline to find the thresholds to apply to the wavelet thresholding algorithm. Two baselines have been considered: 1) Relax task Baseline and 2) Pre-Task Baseline. The proposed algorithm shows better performances than standard Donoho method, in term of noise cancellation and signal distortion, quantified by a new proposed indicator of denoising quality, considering the linear weighted combination of Noise Ratio and Normalized Signal Distortion. Future works will be done in elaborating algorithms that could estimate the thresholds continuously during the entire exercise, keeping always updated the information of the baseline.

\section{ACKNOWLEGMENTS}

This research has been supported by the G-COE Global Robot Academia Program in Waseda University, Japan and partially by a Grant by STMicroelectronics. This research has been conducted at Humanoid Robotics Institute, in collaboration with the G-COE Global Robot Academia. The authors would like to express their gratitude to Okino Industries LTD, Japan ROBOTECH LTD, SolidWorks Corp, Dyden, for their support to the research. Eventually, the authors would like to thank the 10 volunteers who kindly accepted to take part in the experiment.

\section{REFERENCES}

1. J.R.P. Cram, G.S. Kasman, and J. Holtz, Introduction to Surface Electromyography, Jones \& Bartlett Publishers, 1998.

2. D. Knudson and J. Blackwell, "Trunk muscle activation in open stance and square stance tennis forehands" International Journal of Sports Medicine, vol. 21, Jul. 2000, pp. 321-324.

3. C. Hernandez, E. Estrada, L. Garcia, G. Sierra, and H. Nazeran, "Traditional sEMG fatigue indicators applied to a real-world sport functional activity: Roundhouse kick" Electronics, Communications and Computer (CONIELECOMP), 2010 20th International Conference on, 2010, pp. 154-158.

4. G.W. Lange, R.A. Hintermeister, T. Schlegel, C.J. Dillman, and J.R. Steadman, "Electromyographic and kinematic analysis of graded treadmill walking and the implications for knee rehabilitation" The Journal of Orthopaedic and Sports Physical Therapy, vol. 23, May 1996, pp. 294-301.

5. W.B. Kibler, A.D. Sciascia, T.L. Uhl, N. Tambay, and T. Cunningham, "Electromyographic Analysis of Specific Exercises for Scapular Control in Early Phases of Shoulder Rehabilitation" The American Journal of Sports Medicine, vol. 36, 2008, pp. 1789 -1798. 
6. M. Gazzoni, "Multichannel surface electromyography in ergonomics: Potentialities and limits" Human Factors and Ergonomics in Manufacturing \& Service Industries, vol. 20, Aug. 2010, pp. 255-271.

7. A. Troiano, F. Naddeo, E. Sosso, G. Camarota, R. Merletti, and L. Mesin, “Assessment of force and fatigue in isometric contractions of the upper trapezius muscle by surface EMG signal and perceived exertion scale" Gait \& Posture, vol. 28, 2008, pp. 179-186.

8. M. Zecca, S. Micera, M.C. Carrozza, and P. Dario, "Control of Multifunctional Prosthetic Hands by Processing the Electromyographic Signal" Critical Reviews in Biomedical Engineering, vol. 30, 2002, pp. 459-485.

9. C.J. De Luca, L. Donald Gilmore, M. Kuznetsov, and S.H. Roy, "Filtering the surface EMG signal: Movement artifact and baseline noise contamination" Journal of Biomechanics, vol. 43, 2010, pp. 1573-1579.

10.E. Huigen, A. Peper, and C.A. Grimbergen, "Investigation into the origin of the noise of surface electrodes" Medical \& Biological Engineering \& Computing, vol. 40, 2002, pp. 332-338.

11.J.V. Basmajian and C.D. Luca, Muscles Alive: Their Functions Revealed by Electromyography, Lippincott Williams and Wilkins, 1985.

12. M. Rosas-Orea, M. Hernandez-Diaz, V. Alarcon-Aquino, and L. Guerrero-Ojeda, "A Comparative Simulation Study of Wavelet Based Denoising Algorithms" 15th International Conference on Electronics, Communications and Computers (CONIELECOMP'05), Puebla, Mexico: , pp. 125-130.

13. S. Poornachandra, "Wavelet-based denoising using subband dependent threshold for ECG signals" Digital Signal Processing, vol. 18, Jan. 2008, pp. 49-55.

14. M. Blancovelasco, B. Weng, and K. Barner, "ECG signal denoising and baseline wander correction based on the empirical mode decomposition" Computers in Biology and Medicine, vol. 38, 2008, pp. 1-13.

15.D. Donoho and J.M. Johnstone, "Ideal spatial adaptation by wavelet shrinkage" Biometrika, vol. 81, 1994, pp. 425-455.

16. D. Donoho, "De-noising by soft-thresholding" Information Theory, IEEE Transactions on, vol. 41, 1995, pp. 613-627.

17. A. Phinyomark, C. Limsakul, and P. Phukpattaranont, "A Comparative Study of Wavelet Denoising for Multifunction Myoelectric Control" Proceedings of the 2009 International Conference on Computer and Automation Engineering, IEEE Computer Society, 2009, pp. 21-25.

18. Ching-Fen Jiang and Shou-Long Kuo, "A Comparative Study of Wavelet Denoising of Surface Electromyographic Signals" Engineering in Medicine and Biology Society, 2007. EMBS 2007. 29th Annual International Conference of the IEEE, 2007, pp. 1868-1871.

19. R. Coifman and D. Donoho, Translation-Invariant De-Noising, 1995.

20.I.M. Johnstone and B.W. Silverman, "Wavelet Threshold Estimators for Data with Correlated Noise" Journal of the Royal Statistical Society. Series B (Methodological), vol. 59, 1997, pp. 319351.

21.Xiao-Ping Zhang and M. Desai, "Adaptive denoising based on SURE risk" IEEE Signal Processing Letters, vol. 5, Oct. 1998, pp. 265-267.

22.D.L. Donoho and I.M. Johnstone, "Adapting to Unknown Smoothness via Wavelet Shrinkage" Journal of the American Statistical Association, vol. 90, Dec. 1995, pp. 1200-1224.

23. Shi Zhong and V. Cherkassky, "Image denoising using wavelet thresholding and model selection" Proceedings 2000 International Conference on Image Processing (Cat. No.00CH37101), Vancouver, BC, Canada: , pp. 262-265.

24.Y.Y. Tang, P.C. Yuen, C. Li, and V. Wickerhauser, Eds., Wavelet Analysis and Its Applications, Berlin, Heidelberg: Springer Berlin Heidelberg, 2001.

25. Human Kinetics Journals, "The Use of Surface Electromyography in Biomechanics" Apr. 2010.

26. M. Zecca, S. Sessa, Z. Lin, T. Suzuki, T. Sasaki, K. Itoh, H. Hiseki and A. Takanishi, "Development of the ultra-miniaturized Inertial Measurement Unit WB3 for objective skill analysis and assessment in neurosurgery: preliminary results" Med Image Comput Comput Assist Interv. 2009; 12(Pt 1): $443-50$. 